\title{
Compositional Design and Verification of Communication Protocols, using Labelled Petri Nets
}

\author{
JEAN CHRISTOPHE LLORET \\ VERILOG, 150 rue Nicolas Vauquelin, 31081 Toulouse cedex, \\ PierRe azÉma, FrançoIs Vernadat \\ LAAS-CNRS, 7 Avenue Colonel Roche, F-31077 Toulouse cedex
}

\section{Introduction}

This paper proposes a methodology to specify and verify telecommunication protocols by means of Labelled Predicate Transition nets (LPrT). In this paper, the following two principles are used as guidelines.

Modular Specification The structured system decomposition is based upon communication primitives. The rendez-vous communication paradigm of ISO language LOTOS is extended to multi-gate rendez-vous. Several input/output events may appear on a single transition: Petri Net transitions are labelled by sets of communicating events. Incremental Description A single system is described with respect to several levels of abstraction. Each new abstraction level supplies a more detailed model and new properties are to be verified. Communication by multi-rendez-vous is a first means of abstraction. A second means concerns the data part. To facilitate data abstraction in a LPrT net, logic programming (Prolog) is used as a declarative and prototyping language.

The main contributions of this paper concern the support of former principles. Multi-rendez-vous is introduced in a stepwise approach, from basic semantical models, that is Labelled transition systems (LTS) and Labelled Petri Nets (LPN), to labelled Predicate Transition nets. The labelled Predicate Transition nets provide the highest abstraction level and are the user interface model. This stepwise definition presents the following characteristics:

didactical: Synchronization aspects are studied in the context of labelled Petri Nets. Communication with value passing is only introduced for Labelled Predicate transition nets.

analytical: Verification techniques are based on the analysis of the LPrT model behaviour. A specific technique, so-called projection or service computation, is derived from observational equivalence [Mil80]). The global service results from the composition of sub-net services. A modular design of labelled PrT nets entails a modular verification.

The composition of Labelled Petri Nets is described in Section 2. A value passing mechanism and the parameterization of Petri Nets are illustrated in Section 3 by means of a specific application. 


\section{Composition of multi-event Labelled Petri Nets}

This section introduces Labelled Petri Nets and multi-rendez-vous operator $\left.\right|_{L P N}$ as the core language (no value passing) and the basic composition operator, respectively.

The expressive power of this composition operator results from the use of multi-events actions which enable to specify rendez-vous among several (more than two) transitions, on the same or distinct interaction points. This multiple-rendez-vous is an abstraction with respect to implemented communication mechanisms. In the context of the advocated progressive modelling, multi-rendez-vous enables the design of abstract and very compact models.

\subsection{Multi-events actions}

Communication actions are defined according to the following principles:

events: An event is the most elementary communication unit. Let $\alpha$ be the set of gates, let $V$ be the set of interactions. An event is a couple $(g, v)$ which consists of gate $g$ and interaction $v$. Event $(g, v)$ is denoted $g(v)$. Let gate be the function which returns the gate of an event (gate $(g(v))=g$ ) and let $\mathcal{E}$ be the set of events.

1. Multi-event action: an action describes several communication events that are performed synchronously on different gates. A transition is labelled by an action. Because an action refers to a set of events, the expressiveness is increased with respect to the reference languages CCS [Mil80] and LOTOS in which an action is either a single (observable) event or internal action $i$.

2. A single event per gate that is gate is an unshared resource. A service access point is dedicated to a single entity. Consequently, two distinct events on the same gate do not belong to the same action.

Formally, an action $A$ is a subset of events $(A \subseteq \mathcal{E})$ such that, $\forall e_{1}, e_{2} \in A, e_{1} \neq e_{2} \Rightarrow$ gate $\left(e_{1}\right) \neq$ gate $\left(e_{2}\right)$.

Action $\emptyset$ is denoted $i$ (no event $=$ internal action).

\section{Definition 2.1 Labelled Transition system labelling}

Let proc be a labelled transition system; let $\tau$ be proc transition set. Let $\alpha_{\text {proc }} \subseteq \alpha$ be a subset of gates, let $V$ be the set of interactions.

An event $e$, connected to a gate in $\alpha_{\text {proe }}$, is a couple (gate, interaction) $\subset \alpha_{\text {proc }} \times V . e$ is denoted gate(interaction).

proc labelling is couple $\left(\alpha_{\text {proc }}, l_{\text {proc }}\right)$ where $l_{\text {proc }}$ is the label function. The domain of label function $l_{\text {proc }}$ is transition set $\tau$. The range of function $l$ is the subset of actions constituted by events connected in $\alpha_{\text {proc }}$.

In the sequel, $\mathcal{L}_{\text {proc }}$ denotes couple $\left(\alpha_{\text {proc }}, l_{\text {proc }}\right)$.

\subsection{Multi-rendez-vous Composition of Labelled Transition Systems}

Labelled Transition Systems supply an operational semantics to Labelled Petri Nets. The multi-rendez-vous of labelled transition systems is the interpretation of multirendez-vous of Petri nets. 


\section{Definition 2.2 Labelled Transition System}

A labelled transition system proc is a 5 -tuple $\left(S, T,-t \rightarrow_{t \in T}, s_{0}, \mathcal{L}\right)$ where:

- $S$ set of states.

- $T$ set of transitions.

- $t \rightarrow \subset S \times S$ state change performed by transition $t$.

- $s_{0} \in S$, initial state.

- $\mathcal{L}$ is a labelling as defined above.

Two labelled Transition systems proc 1 and proc $_{2}$ are composed relative to their common gates.

LTS common gates: the set of common gates $\alpha_{\text {proc }} \cap \alpha_{\text {proc }}$ is denoted $\alpha_{n}$. events to be synchronized: events to be synchronized of label $l(t)$ are events whose gate is a shared gate between proc $c_{1}$ and proc 2 . Notation is $\operatorname{sync}_{\alpha_{n}}(l(t))$ :

sync $_{\alpha_{n}}(l(t))=\left\{e \in l(t) \mid\right.$ gate $\left.(e) \in \alpha_{n}\right\}$.

Definition 2.3 LTS composition operator $\mid L T S$ Let proc $_{i}=\left(S_{i}, \tau_{i},-t_{i} \rightarrow \rightarrow_{i} \in \tau_{i}\right.$ $\left., s_{i, 0}, \mathcal{L}_{i}\right)_{i=1,2}$ be two LTS.

Composed LTS proc $\mid$ Lrs proc $_{2}$ is $\left(S_{1}\left|S_{2}, T_{1}\right| T_{2},-t \rightarrow t \in T_{1}\left|\tau_{2}, s_{1,0}\right| s_{2,0}, \mathcal{L}_{1} \mid \mathcal{L}_{2}\right)$ where:

$\mathcal{L}_{1} \mid \mathcal{L}_{2}$ is proc $_{1} \mid$ proc $_{2}$ labelling defined by set of gates $\left(\alpha_{\text {proc }} \cup \alpha_{\text {proc }}\right)$ and labelling function $l$ defined on domain $T_{1} \mid T_{2}$.

Function $l$ is defined together with composed states and composed transitions by the following derivation rules ( $s_{i}, s_{i}^{\prime}$ and $t_{i}$ are states and a transition of proc $c_{i}$ respectively):

Independant execution

$$
\begin{aligned}
& \text { (1) } \frac{s_{1}-t_{1}: l_{1} \rightarrow s_{1}^{\prime}}{s_{1}\left|s_{2}-t_{1}: l_{1} \rightarrow s_{1}^{\prime}\right| s_{2}}\left(\operatorname{sync}_{\alpha_{n}}\left(l_{1}\right)=\emptyset\right) \\
& \text { (2) } \frac{s_{2}-t_{2}: l_{2} \rightarrow s_{2}^{\prime}}{s_{1}\left|s_{2}-t_{2}: l_{2} \rightarrow s_{1}\right| s_{2}^{\prime}}\left(\operatorname{sync}_{\alpha_{n}}\left(l_{2}\right)=\emptyset\right)
\end{aligned}
$$

Synchronization

$$
\text { (3) } \frac{s_{1}-t_{1}: l_{1} \rightarrow s_{1}^{\prime}, s_{2}-t_{2}: l_{2} \rightarrow s_{2}^{\prime}}{s_{1}\left|s_{2}-t_{1}\right| t_{2}:\left(l_{1} \cup l_{2}\right) \rightarrow s_{1}^{\prime} \mid s_{2}^{\prime}}\left(\operatorname{sync}_{\alpha_{n}}\left(l_{1}\right)=\operatorname{sync}_{\alpha_{n}}\left(l_{2}\right)\right)
$$

\subsection{Labelled Petri Nets}

Firstly, Labelled Petri Nets are defined as Place/Transition nets of capacity one, associated with a labelling as defined above.

The behaviour of a labelled Petri Nets is then introduced as a labelled transition system. The marking graph and the step graph are two possible candidates for describing a net behaviour. In the step graph, parallel executions are explicitly taken into account and thus a more precise representation of the net behaviour is given. Labelled Petri Net operator $\left.\right|_{L P N}$ is interpreted on step graphs by Labelled Transition System operator lLTs in such a way that diagram 1 commutes. 


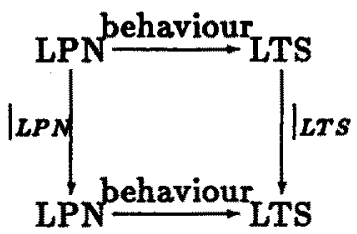

LPN: Labelled Petri Nets

LTS: Labelled Transition Systems

$B(N)$ : net $N$ behaviour (step graph)

$\forall N_{1}, N_{2} \in L P N, B\left(\left.N_{1}\right|_{L P N} N_{2}\right)=\left.B\left(N_{1}\right)\right|_{L T S} B\left(N_{2}\right)$

Figure 1: Semantics of Labelled Petri Nets composition " $\left.\right|_{L P N}$ "

\section{Definition 2.4 Labelled Petri Net}

A labelled Petri Net is a 6-tuple $N=\left(P, \tau\right.$, pre, post, $\left.M_{0}, \mathcal{L}_{N}\right)$ where:

- $P$ is a set of places;

- $T$ is a set of transitions, $P \cap T=\emptyset$

- pre, post : $\tau \rightarrow P(P)$ are two mappings which connect a transition to two sets of places called preconditions and postconditions respectively.

- $M_{0}$ is a set of places called initial marking.

- $\mathcal{L}_{N}$ is the net labelling defined by couple $\left(\alpha_{N}, l_{N}\right)$ as introduced previously.

Example: net $N_{1}$, Fig 2, depicts concurrent transitions $t_{1}$ and $t_{2}$ (places are circles, transition boxes inscribed by a transition name; if a transition box is connected to a gate, the edge is annoted by the value of the corresponding transition event; the preconditions and postconditions of a transition are the input and output places respectively; places with a token belong to the initial marking).

Definition 2.5 Firing rule The transition firing rule proposed for "augmented condition/event nets" in [PD87] is adopted. But the definition of parallel transitions differs because an interaction point is a non shared resource: with respect to labelled Petri nets, two transitions connected to a common interaction point cannot be fired in parallel.

Let $M$ be a marking (or set of places) and $T$ a set of transitions (set $T$ is called step). $T$ is $M$ enabled (notation $M[T>$ ) iff

- $T$ transitions are pairwise independent that is two distinct transitions $t_{1}, t_{2} \in T$ share neither a place (place independance: $\left(p r e\left(t_{1}\right) \cup\right.$ post $\left.\left(t_{1}\right)\right) \cap\left(p r e\left(t_{2}\right) \cup\right.$ post $\left.\left.\left(t_{2}\right)\right)=\emptyset\right)$ nor a gate (gate independance: gate $\left(l_{N}\left(t_{1}\right)\right) \cap$ gate $\left(l_{N}\left(t_{2}\right)\right)=\emptyset$, where gate $\left(l_{N}(t)\right)=\{$ gate $(e) \mid$ $\left.\left.e \in l_{N}(t)\right\}\right)$.

- preconditions of $T$ transitions are fulfilled $\left(\operatorname{pre}(T) \subset M\right.$, with pre $(T)=U_{t \in T}$ pre $\left.(t)\right)$ and postconditions which are not preconditions are not fulfilled $(M \cap(p o s t(T) \backslash p r e(T))=$ ๑).

Marking $M^{\prime}$ results from firing step $T$ in marking $M$ (notation $M\left[T>M^{\prime}\right.$ ) iff step $T$ is enabled in $M$ and $M^{\prime}=(M \backslash p r e(T)) \cup \operatorname{post}(T)$.

The behaviour of a LPN is a LTS such that a state is a reachable marking and a LTS transition is a firable step.

\section{Definition 2.6 $L P N$ Behaviour}

Let $N=\left(P, \tau\right.$, pre, post, $\left.M_{0}, \mathcal{L}_{N}\right)$ be a LPN. Reachable markings and firable steps of net $N$ are respectively the smaller sets $M_{0} \mid>$ and $M_{0} \mid$ defined by:

- $M_{0} \in\left[M_{0}>\right.$

- If $M \in\left[M_{0}>\right.$ and $T$ enabled in $M$ with $M \mid T>M^{\prime}$ then $M^{\prime} \in M_{0} \mid>$ and $T \in M_{0} \mid$. 
Net behaviour is LTS, $B(N)=\left(M_{0}\left[>, M_{0}\left[,-T \rightarrow_{T \in M_{0}[}, M_{0}, \mathcal{L}_{B}\right)\right.\right.$ where:

- change of state in $B(N)$ corresponds to step execution: $M, M^{\prime} \in M_{0} \mid>, M-T \rightarrow M^{\prime} \Leftrightarrow$ $M\left[T>M^{\prime}\right.$.

- Behaviour labelling $\mathcal{L}_{B}=\left(\alpha_{B}, l_{B}\right)$ directly follows net labelling: the gate set of $\mathcal{L}_{B}$ is $\mathcal{L}_{N}$ one, i.e. $\alpha_{B}=\alpha_{N}$. Labelling function $l_{B}$ is the canonical extension to sets of the net transition labelling function $l_{N}: \forall T \in M_{0}\left[, l_{B}(T)=\bigcup_{t \in T} l_{N}(t)\right.$.

(a)

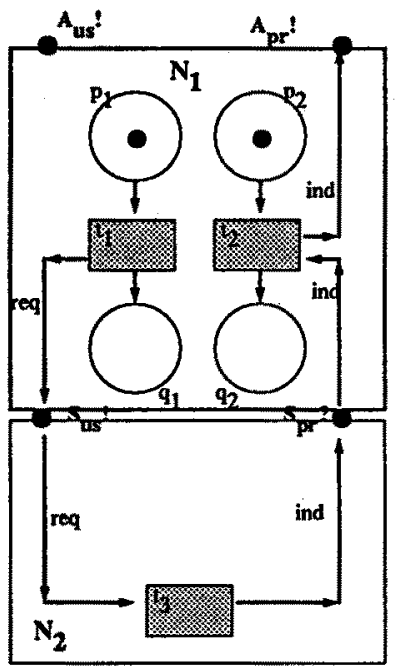

(b)

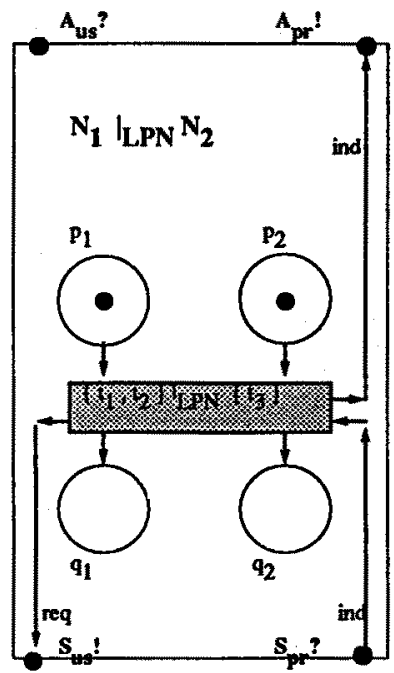

Figure 2: Parallel composition of Labelled Petri Nets

\subsection{Multi-rendez-vous Composition of Labelled Petri Nets}

Operator $\left.\right|_{L P N}$ is defined in order to simulate composition of behaviours. The three following characteristics of net multi-rendez-vous are worth noticing:

1. Parallelism between transitions is taken into account When composing two labelled Petri nets, two transitions of a net, which fire in parallel, may be merged with a transition of the other net (on Fig 2 transition $\left.\left\{t_{1}, t_{2}\right\}\right|_{L P N}\left\{t_{3}\right\}$ results from the merging of transition sets $\left\{t_{1}, t_{2}\right\}$ and $\left.\left\{t_{3}\right\}\right)$.

2. Minimal mergings The possibility of running synchronously (transitions are in parallel) is distinguished from a necessary synchronization (transitions are merged). In the composition of two nets, all synchronization possibilities are preserved but the number of mergings is minimal.

3. The derivation of a composed net $\left.N_{1}\right|_{L P N} N_{2}$ does not require computation of behaviours $B\left(N_{1}\right)$ and $B\left(N_{2}\right)$.

Let $N_{i}=\left(P_{i}, \tau_{i}, \text { pre }_{i}, \text { post }_{i}, M_{0, i}, \mathcal{L}_{i}\right)_{i=1,2}$ be two LPN with labelling $\mathcal{L}_{i}=\left(\alpha_{i}, l_{i}\right)_{i=1,2}$ and let $\alpha_{n}$ be the common gate alphabet.

Preliminary definitions:

Transition to be merged: Transition $t$ of net $N_{1}$ or $N_{2}$ is to be merged iff there is at least one event to be synchronized in $t$ label (recall that an event to be synchronized is 
an event whose gate belongs to common gate set $\alpha_{n}$ ).

Mergeable Transition sets: Two transition sets $T_{1} \subseteq T_{1}$ and $T_{2} \subseteq T_{2}$ are mergeable iff $T_{1}$ (resp. $T_{2}$ ) is a set of transitions to be merged, pairwise independent and the set of events to be synchronized of $T_{1}$ transitions equals the one of $T_{2}$ transitions (i.e. $\left.\operatorname{sync}_{\alpha_{n}}\left(l_{1}\left(T_{1}\right)\right)=\operatorname{sync}_{\alpha_{n}}\left(l_{2}\left(T_{2}\right)\right)\right)$.

Minimal mergeable Transition sets: $T_{1}$ and $T_{2}$ are minimal mergeable transition sets iff $T_{1}$ are mergeable transition sets and if it is not possible to partition $T_{1}, T_{2}$ merging in smaller ones that is: for all $T_{1}^{\prime} \subset T_{1}$ and $T_{2}^{\prime} \subset T_{2}, T_{1}^{\prime}$ and $T_{2}^{\prime}$ are not mergeable.

\section{Definition 2.7 LPN composition operator $\left.\right|_{L P N}$}

Composed net $\left.N_{1}\right|_{L P N} N_{2}$ is $\left(P_{1} \cup P_{2},\left.T_{1}\right|_{L P N} T_{2}\right.$, pre, post, $\left.M_{1,0} \cup M_{2,0},\left.\mathcal{L}_{1}\right|_{L P N} \mathcal{L}_{2}\right)$ where:

- $\left.T_{1}\right|_{L P N} T_{2}$ transitions are, on the one hand, $T_{1}$ and $T_{2}$ transitions which are not to be merged and, on the other, merged transitions $\left.T_{1}\right|_{L P N} T_{2}$ where $T_{1}, T_{2}$ are minimal mergeable transition sets.

- Preconditions and postconditions of a nonmerged transition are unchanged in composed net. Pre and postconditions of merged transition $\left.T_{1}\right|_{L P N} T_{2}$ are defined as the set union of the respective pre and postconditions: pre $\left(\left.T_{1}\right|_{L P N} T_{2}\right)=\operatorname{pre}_{1}\left(T_{1}\right) \cup$ pre $2\left(T_{2}\right)$ and similarly for postconditions.

- New labelling $\left.\mathcal{L}_{1}\right|_{L P N} \mathcal{L}_{2}$ : gate sets of the composed nets are added; the labelling of a nonmerged transition is unchanged; labelling of merged transition $\left.T_{1}\right|_{L P N} T_{2}$ is $l_{1}\left(T_{1}\right) \cup l_{2}\left(T_{2}\right)$.

Example: figure $2 \mathrm{~b}$ depicts composed net $\left.N_{1}\right|_{L P N} N_{2}$.

\section{Proposition 2.1 Properties of multi-rendez-vous composition}

Operators $\left.\right|_{L T S}$ and $\left.\right|_{L P N}$ are commutative and associative (up to state and transition bijective renaming).

The behaviour of a composed net is the composition of behaviours: $B\left(\left.N_{1}\right|_{L P N} N_{2}\right)=$ $\left.B\left(N_{1}\right)\right|_{\text {Lrs }} B\left(N_{2}\right)$ (up to state and transition bijective renaming).

\section{Application}

This section introduces Labelled Predicate Nets by means of an example. LPrT nets are a parameterized version of LPN; they are also PrT nets [Gen88] extended with labels, and featuring, in particular, direct execution in Prolog.

A remote reading mechanism, the so-called Telereport [HRJ89] application layer, is first modelled then analysed.

\subsection{Models}

Application entities $A_{u s}$ and $A_{p r}$ cooperate through a nonperfect session service $S$ (see Fig 3 ) in order to provide a remote reading service to a user process.

Application user service. The interface between the user process and entity $A_{u s}$ is of particular interest since it determines the external service provided to the user process: user request req $(C)$ is parameterized by requested data code $C$ and is issued on Service Access Point, $A_{u s}$ ?; confirmation conf $(M e s s)$ is issued on SAP $A_{u s} !$ and can 
be of 3 types: Mess value is either "error": transmission by session service has failed, or "nak": no data of code $C$ is available to the provider process, or $C$ read value $V a l_{C}$. Entity $A_{u}$ is in charge of recovery if a transmission error occurs in the session service. Let $N R_{M a x}$ be the maximum number of consecutive recoveries with respect to the same read request. To study the correctness of the recovery mechanism a specific model of session service has been designed: session service may lose consecutively at most $N E_{M a x}$ messages. The provided service depends on relative values of parameters $N R_{M a x}$ and $N E_{\text {Max }}$.

Configuration. Three configuration classes are distinguished: (1) Perfect session service (no error $N E_{\max }=0$ ); (2) Faulty session service but less errors than recoveries ( $N E_{\max } \leq N R_{\text {max }}$ ); (3) Unreliable service: more errors than recoveries ( $\left.N E_{\max }>N R_{\max }\right)$. Furthermore, the requested code may either be available to the provider (expected confirmation with value) or not (expected confirmation: "nakn). The set of potential configurations, and the database facts are the following:

\begin{tabular}{|l|c|c|l|l|}
\hline & $\operatorname{con} f_{0}$ & $\operatorname{con} f_{1}$ & $\operatorname{con} f_{2}$ & database \\
\hline$N E_{M a x}$ & 0 & $\operatorname{succ}(0)$ & $\operatorname{succ}(\operatorname{succ}(0))$ & $M a x E r r\left(N E_{\max }\right)$. \\
\hline$N R_{\max }$ & \multicolumn{3}{|c|}{$\operatorname{succ}(0)$} & $M a x \operatorname{Recover}(\operatorname{succ}(0))$. \\
\hline $\operatorname{code} c_{1}$ & \multicolumn{3}{|c|}{$c_{1}$ available $\rightarrow$ value $v_{1}$} & $\operatorname{codeT} y p e\left(c_{1}\right) \cdot \operatorname{codeV} a l\left(c_{1}, v_{1}\right)$. \\
\hline $\operatorname{code} c_{2}$ & \multicolumn{3}{|c|}{$c_{2}$ not available $\rightarrow$ nak } & $\operatorname{codeType}\left(c_{2}\right)$. \\
\hline
\end{tabular}

Session Service Net (see Fig 3, net $S$ )

A request $\operatorname{req}(C)$ or a response resp(Mess) may be conveyed without error from user entity $A_{u}$ to provider entity $A_{\mathrm{pr}}$ respectively and vice-versa. Primitive reg becomes ind and resp becomes conf. The corresponding normal transitions are transmit Reg and transmitResp. Request $\operatorname{req}(C)$ or response resp(Mess) may either be lost or incorrectly transmitted. This corresponds to transitions loseReg and loseResp. The number of consecutive errors before a reset signal (transition reset) is saved by variable $K$ and bounded by logical condition infMaxErr $(K)$. Predicate $\operatorname{in} f \operatorname{Max} \operatorname{Err}(K)$ is defined by the following clause:

$/^{*}$ infMaxErr $(K)$ is true if $K<N E_{\max } *$ /

infMax $\operatorname{Err}(K):-\max \operatorname{Err}\left(N E_{\max }\right), /^{*}$ database fact */

$$
\text { in } f\left(K, N E_{M a x}\right) . \quad / * \text { true if } K<N E_{\text {max }} * /
$$

The occurrence of an error results in sending message conf(error) to user entity $A_{u}$ o (transition error).

User entity of Application protocol (see Fig 3, net $A_{u s}$ )

The normal transition sequence is (1) init receipt of user process request reg $(C),(2)$ send request transmission to session service, (3) end confirmation conf(Mess) is received and returned to the user process, if Mess $\neq$ error, that is Mess is a code value or "nak"). When a session service error is detected, via primitive conf(error)), recovery may take place, i.e. user process request is repeated. Transition recover is enabled if less than $N R_{\max }$ errors have occured; this enabling condition, infMaxRecover $(K)$, is defined by the following clause:

infMaxRecover $(K):-M a x R e c o v e r\left(N R_{\text {max }}\right)$

$$
\text { inf }\left(K, N R_{\max }\right) \text {. }
$$


In case of confirmation conf(error) and if the maximum number of recoveries is exceeded, transition end fires. Signal reset is sent to session service.

Provider entity of Application protocol (see Fig 3, net $A_{p r}$ )

Provider entity is initially ready to receive read indication ind $(C)$ : transition indication is enable; procedure readCode $(C, \mathrm{ValOrNak})$ of transition response performs a read action; when $C$ is available (codeVal $(C, V a l)$ in database) the value of $V$ al is substituted for variable $\mathrm{ValOrNak}$; if $C$ is not available variable $V$ alOrNak takes value nak, negative acknowledge. Procedure readCode is defined by the following net clause:

readCode $(C, V a l):-\operatorname{codeV} a l(C, V a l)$. code available

readCode $(C, n a k):-1+\operatorname{codeV} a l(C, V a l)$. code not available

\subsection{Verification}

An "easy to use" verification technique is to compute the service provided by the protocol and to compare it with the expected one.

The global service provided by a behaviour (LTS) is a reduced LTS, minimal with respect to the state number, which preserves properties of observed communication actions; with respect to gate set $G$, a local service is derived from the behaviour after the hiding of events which occur on gates outside $G$. The standard observational equivalence [Mil80] is used to compute the service; observable actions sequences and deadlocks are preserved.

\section{Service Derivation}

Let $N$ be a LPN system composed of three sub-nets: $N=\left.\left.N_{1}\right|_{L P N} N_{2}\right|_{L P N} N_{3}$. Net service $S(N)$ is defined as the service provided by the behaviour of $N$, i.e. $S\left(B_{L P N}(N)\right)$. There are two basic ways to compute service $S(N)$; a third one may also be derived in a combined manner:

Service composition: individual subnet services are computed first, then they are composed. We have, $\left.\left.S(N) \approx S\left(N_{1}\right)\right|_{\text {LTS }} S\left(N_{2}\right)\right|_{\text {LTS }} S\left(N_{3}\right)$. Partial verifications may be conducted on individual services, and then reused for the verification of the global service. Furthermore, as services are reduced behaviours, composition of individual services can be more efficient than computation of the global netbehaviour.

$L P N$ composition sub-nets are composed to obtain a global net whose behaviour and service are derived in a second step. This approach is mandatory when individual behaviours are unbounded, even if the global behaviour is bounded. For example, in case of PrT nets, unbounded net may be a fifo queue model.

Combined approach: The former computations produce an equivalent result because diagram 1 commutes and because Milner observational equivalence $\approx$ is a congruence with respect to $\left.\right|_{\text {LrS }}$. A combined approach may still be useful to gain a better insight into protocols. For example, $\left.S(N) \approx S\left(\left.N_{1}\right|_{L P N} N_{2}\right)\right|_{L T S} S\left(N_{3}\right)$ : on the one hand $N_{3}$ individual service is computed, and on the other net composition $\left.N_{1}\right|_{L P N} N_{2}$ may be performed.

Telereport Service Local service provided to user process is depicted in Fig 4 (transitions represent application service primitives). As long as errors are relatively few, i.e., 


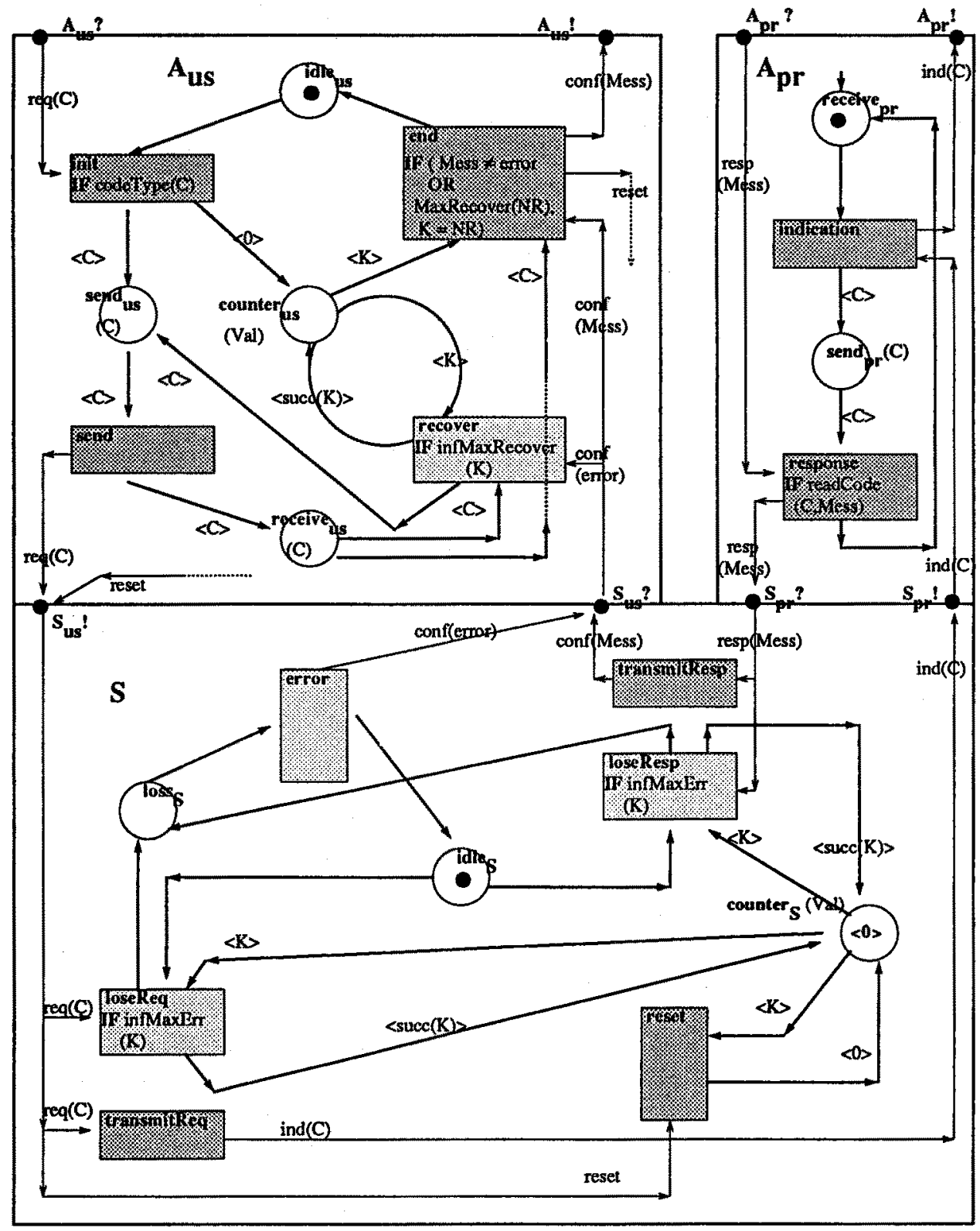

Figure 3: LPrT nets of Application entities $A_{u s}$ and $A_{p r}$ and session service $S$ 
in configurations con $f_{0}, \operatorname{con} f_{1}$, the provided service is error-free: the recovery mechanism plays the intended role. Configuration $c o n f_{3}$ is a degraded configuration: errors may be too numerous, and the application service may not deliver the expected answer $\left(v_{1}\right.$ or nak). Correct status error is however delivered to user process. Confirmation conf(error) follows an internal transition after the last (unrecovered) error.
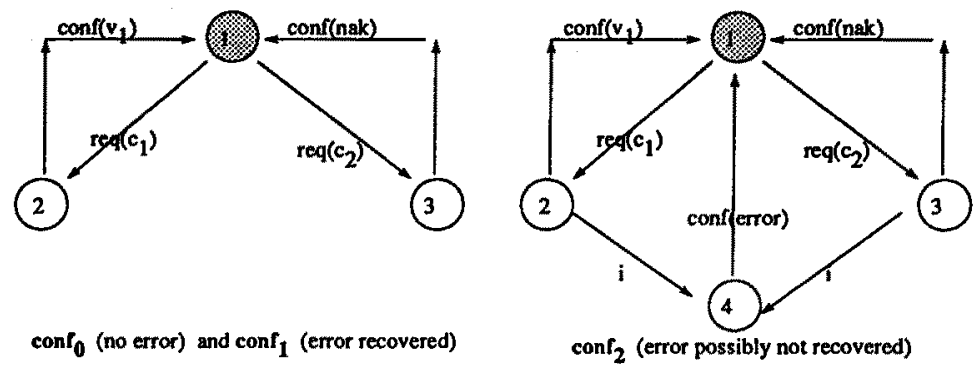

Figure 4: Application service with respect to user process

\section{Conclusion}

The introduced composition and verification techniques are implemented in the available software PIPN. The tool enforces structured and incremental approaches. It includes a graphical SADT-like editor that defines system architecture. The compiler computes global nets, as a result of subnet composition. State space can be interactively explored by means of the simulator. Finally, the complete behaviour can be computed and then reduced to protocol service. A model checker for CTL logic is also available. A current issue is to combine logic and service verification in a combined approach [PA89].

\section{References}

[Gen88] Hartmann J. Genrich. Equivalence transformation of PrT-nets. In Workshop on Application and Theory of Petri Nets, pages 229-248, 1988.

[HRJ89] P. Haudebourg, G. Revelaud, and T. Journey. Spécification du protocole de téléreport. Technical Report HR/23-2077 and HR23-1943, Electricite De France, DER, Clamart, 1989.

[Mil80] Robin Milner. A Calculus of Communicating Systems, volume 92 of Lecture Notes in Computer Science. Springer-Verlag, 1980.

[PA89] F. Vernadat P. Azema, J.C. Lloret. Requirement analysis for communication protocols. In Workshop On Automatic Verification Methods for Finite State Systems, GrenobleFrance, 1989.

[PD87] U. Montanari P. Degano, R. De Nicola. A distributed operational semantics fot ccs based on condition/event systems. Technical Report 111, Instituto di Elaboratione dell 'Informazione, C.C.R, Pisa, 1987. 